\title{
Cervix carcinoma and incidental finding of medullary thyroid carcinoma by 18F-FDG PET/CT — clinical case
}

Borislav Chaushev ${ }^{1}$ Pavel Bochev¹, Anelia Klisarova1', Kaloyan Yordanov¹, Elitsa Encheva1', Jivka Dancheva', Cvetelina Yordanova1, Kiril Hristozov², Ivan Krasnaliev³, Radoslav Radev, Rumen Nenkov4

Department of Nuclear Medicine and Metabolic Therapy ${ }^{1}$, Department of Endocrinology ${ }^{2}$, Department of Pathoanatomy ${ }^{3}$, Thoracic Surgery4, MBAL "St. Marina", Varna, Bulgaria

The authors declare that they have no conflicts of interest.

[Received 6 III 2014; Accepted 7 VII 2014]

\begin{abstract}
Thyroid nodules are encountered in clinical practice during the diagnostic procedures or patients' follow-up due to other diseases quite far from the thyroid gland with prevalence $4-50 \%$ in general population, depending on age, diagnostic method and race. The prevalence of thyroid nodules increases with age and their clarification should be done for their adequate treatment. An 18F-FDG PET/CT was done with a PET/CT scanner (Philips Gemini TF), consisting of dedicated lutetium orthosilicate full ring PET scanner and 16 slice CT.

The PET/CT scan of the whole-body revealed on the CT portion a hypodense nodular lesion in the left lobe of the thyroid gland with increased uptake of $18 \mathrm{~F}-F D G$ on the PET with SUV ${ }_{\max } 10.3$ and demonstrated a complete response to the induction therapy of the main oncological disease of the patient - squamous cell carcinoma.

This clinical case demonstrates that whole-body 18F-FDG-PET/CT has an increasingly important role in the early evaluation of thyroid cancer as a second independent malignant localization. Focal thyroid lesion with high risk of thyroid malignancy was incidentally found on 18F-FDG PET/CT.
\end{abstract}

KEY words: PET/CT, 18F-FDG, hypodense nodular lesion, medullary thyroid cancer

Nuclear Med Rev 2014; 17, 2: 97-100

\section{Introduction}

Asymptomatic thyroid nodules are often encountered in clinical practice and its prevalence is $4-50 \%$ in general population depending on age, diagnostic methods and race. The prevalence of thyroid nodules increases with age. Thyroid incidentalomas are characterized as focal not palpable intrathyroidal new nodular lesions detected by imaging modalities during study for non-thyroidal disease. These nodules are usually not palpable and benign, with an associated risk of cancer ranging from 1.5-10\% [1, 2].

Positron emission tomography (PET/CT) using 18F-fluorodeoxyglucose (FDG) is increasingly performed for staging and for exact

Correspondence to: Borislav Chaushev, MD, PhD,

Specialist of Nuclear Medicine

Department of Nuclear Medicine and Metabolic Therapy

MBAL "St. Marina", Varna, Bulgaria

E-mail: bchaushev@gmail.com localization of metastatic disease in patients with various kinds of malignancies [2-5]. The FDG PET/CT provides the advantages of the two modalities; the anatomic information is provided by the spiral CT and the functional information by the FDG PET. This combined approach has resulted in a significant improvement in both anatomic localization and diagnostic accuracy $[6,7]$.

The uptake of FDG in the normal thyroid gland is usually not visualized on FDG-PET [8, 9].

Focal or diffuse FDG uptake in the thyroid gland could be seen as an incidental finding. Some studies have reported that the incidence of thyroid incidentalomas with increased FDG uptake is $1.2-2.3 \%$ on PET examinations $[7,8,10,11]$.

Subject of our study was to evaluate treatment response with a whole-body 18F-FDG PET/CT in a 61 year old female with squamous cell cervix carcinoma, pT2pN1M0. After the induction therapy, radical laparohysterectomy with colpo-urethro-cystectomy, lymphadenectomy bilateral in the pelvis, para-aortal and presacral lymph nodes, chemotherapy and radiotherapy were performed. 


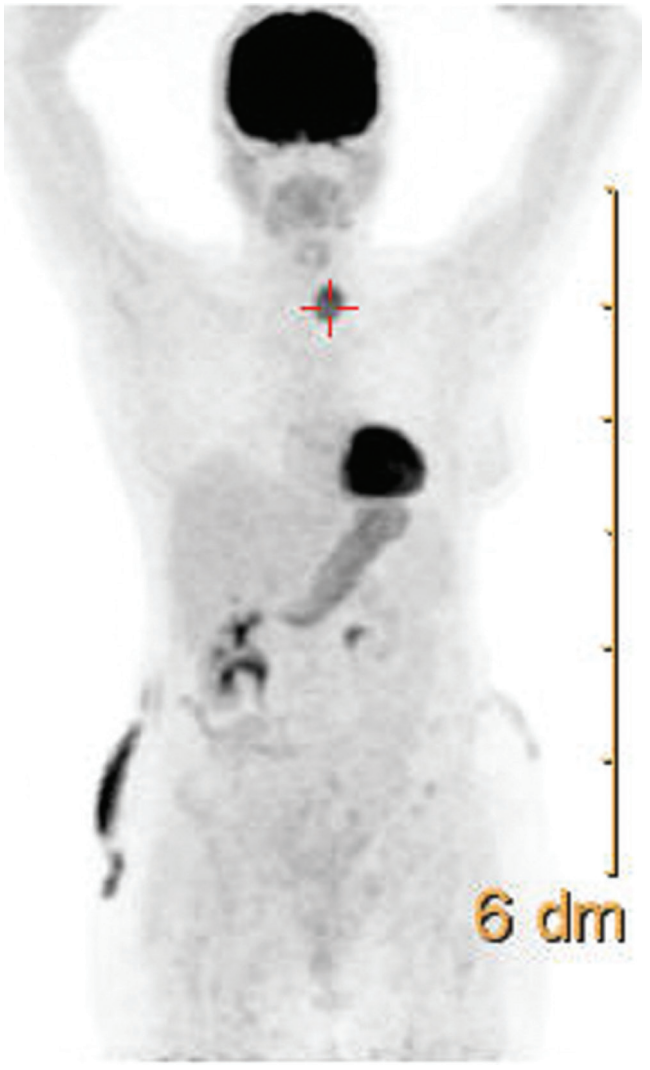

Figure 1. Physiologic uptake of the 18F-FDG-PET true-out the body in the heart, right kidney after the surgery and hypermetabolic increased uptake of the 18F-FDG in the left anterior part of the neck base

\section{Case report}

The whole-body 18F-FDG PET/CT images ware done applying a PET/CT scanner (Philips Gemini TF), consisting of dedicated lutetium orthosilicate full ring PET scanner and 16 slice CT. Standard patient preparation included at least 6 hours fasting and serum glucose level of less than $120 \mathrm{mg} / \mathrm{dl}$ before 18F-FDG administration. $\mathrm{PET} / \mathrm{CT}$ imaging was performed 60 minute after intravenous injection of $370 \mathrm{MBq}(10 \mathrm{mCi})$ of $18 \mathrm{~F}-\mathrm{FDG}$.

At 60 minute after administration of $18 \mathrm{~F}-F D G$, low dose CT (50 mAs, $120 \mathrm{kV}$ ) covering area from scull to the proximal thighs was performed for the purpose of attenuation correction and precise anatomical localization. Thereafter, emission scan was conducted in the three-dimensional mode. Emission scan time per $\mathrm{mm}$ was $39 \mathrm{~mm} / \mathrm{sec}$. PET data were obtained using a high-resolution whole-body scanner with an axial field of view of $57.6 \mathrm{~cm}$. The average total PET/CT examination time was $20 \mathrm{~min}-$ utes. Standardized uptake value $\left(S_{U} U V_{\text {max }}\right)$ was estimated for every hypermetabolic lesion.

After scatter and decay correction, PET data were reconstructed iteratively with attenuation correction and they were reoriented in axial, sagittal, and coronal slices. The row action maximum-likelihood algorithm was used for three dimensional reconstructions.
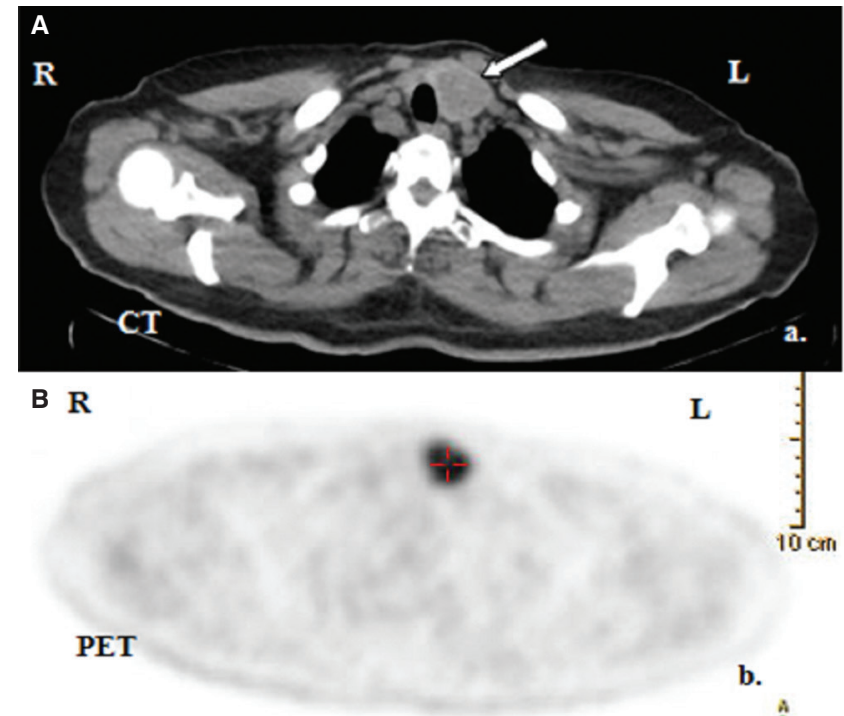

A

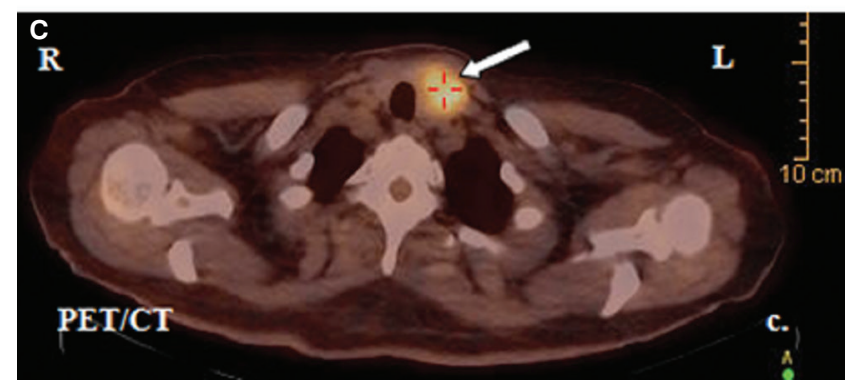

Figure 2A-C. Transaxial scan of the CT, PET and PET/CT.

A. Hypodense nodular lesion in the left lobe of the thyroid gland (white arrow); B. Hypermetabolic increased uptake of the 18F-FDG in the level of the left-anterior part of the base of the neck without exact anatomical localization; C. Focal FDG uptake with exact anatomical localization in the left lobe of the thyroid gland on PET/CT (white arrow). Histopathological data after a FNAB under an US control verified medullar thyroid carcinoma

\section{Discussion}

The PET/CT whole-body scan revealed loco-regional complete therapeutic response of the squamous cell cervix carcinoma (Figure 1) and a hypodense nodular lesion in the left lobe of the thyroid gland

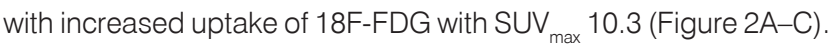
After the PET/CT scan of the whole body, fine needle aspiration biopsy (FNAB) was performed of the left lobe of the thyroid gland nodular lesion under ultrasound (US) control.

The result of the biopsy showed a thyroid tumor with lobular pattern. The stroma of the tumor is composed of fibrovascular tissue. Tumor cells are polygonal or slightly spindled without conspicuous nucleoli and with granular chromatin. $\mathrm{ICH}$-positive immunoreactivity for synaptophysin and TTF1.

Patient underwent thyroidectomy. Histopathological diagnosis was - medullary carcinoma of thyroid (Figure 3). After the surgery the thyrocalcitonin was normal but during the patient follow-up increased and in the sixth month reached $1832 \mathrm{pg} / \mathrm{ml}$ (ref. 0-13) and a control whole-body 18F-PET/CT was done. 


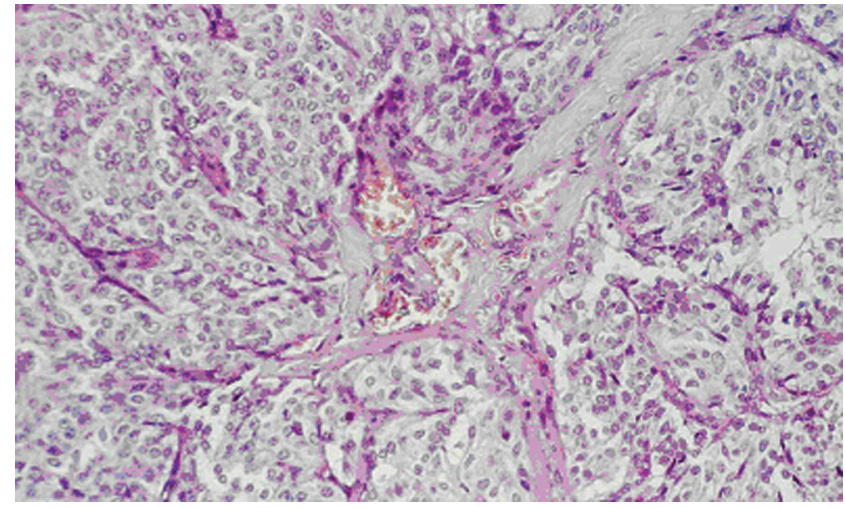

Figure 3. Fine needle aspiration biopsy - $\mathrm{ICH}$-positive immunoreactivity for synaptophysin and TTF1. Diagnosis - medullary carcinoma of thyroid
On the 18F-FDG-PET hypermetabolic multiple pulmonary, bone and soft tissue metastases were visualized (Figure 4). PET/CT demonstrated a progression of the medullary carcinoma disease with the whole body aggressive metastatic involvement.

\section{Conclusion}

The current widespread usage of whole-body $18 \mathrm{~F}-\mathrm{FDG}$ $\mathrm{PET} / \mathrm{CT}$ studies as a screening tool for evaluation of malignant tumors has resulted in an increase in the detection of incidentally found thyroid lesions.

In fact, as this technology becomes more available, the incidence of thyroid incidentalomas will likely continue to increase. Focal thyroid lesions incidentally found on 18F-FDG PET/CT have high risk of thyroid malignancies.

Whole-body 18F-FDG PET/CT is playing an increasingly important role in the evaluation of asymptomatic thyroid cancer.

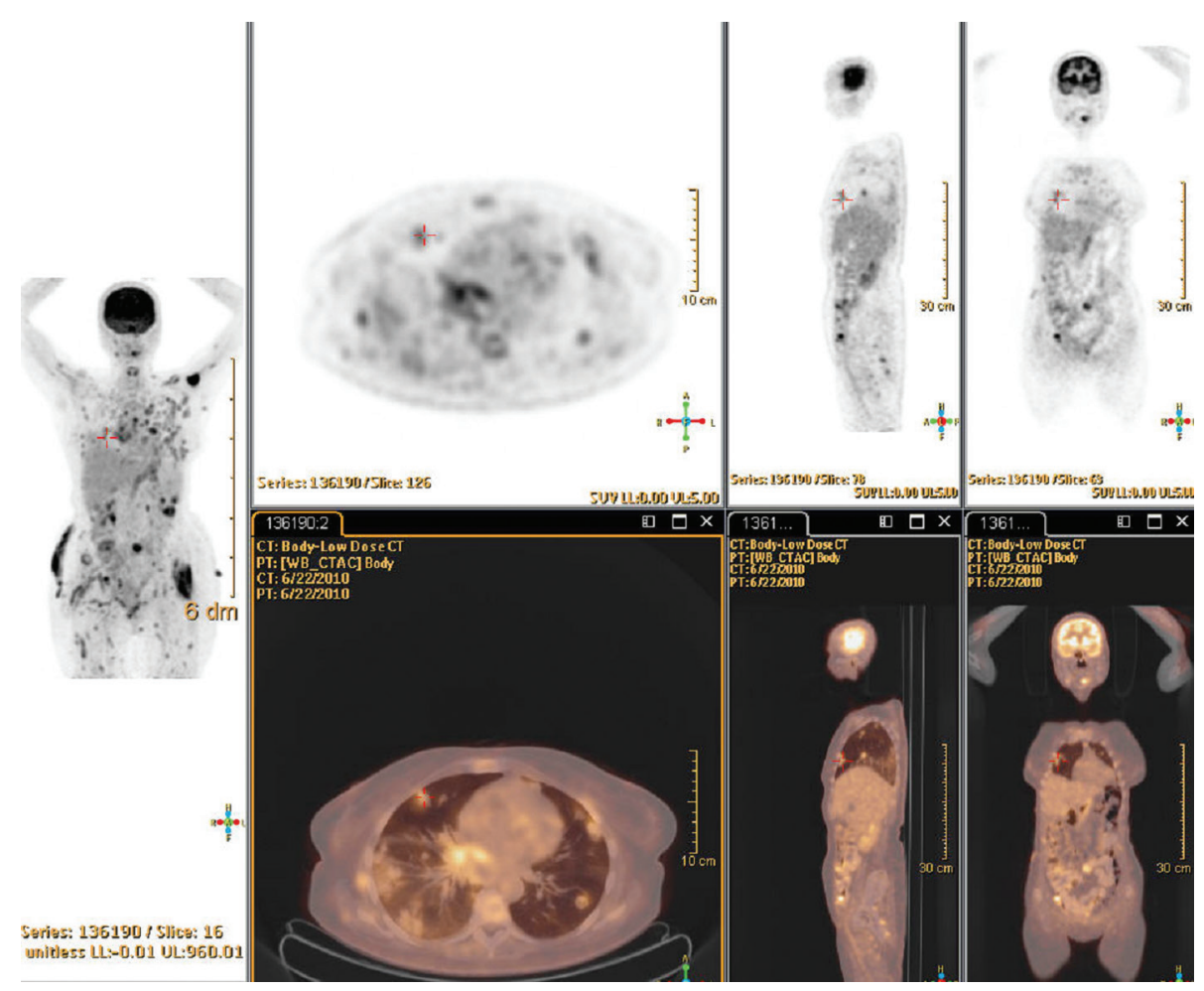

Figure 4. 18F-FDG PET/CT whole-body scan displays multiple pulmonary, bone and soft tissue metastases and a disease progression

\section{References}

1. Siegel R, Naishadham D, Jemal A. Cancer statistics, 2012. CA Cancer J Clin 2012; 62: 10-29.

2. Pelosi E, Messa C, Sironi S et al. Eur J Nucl Med Mol Imaging 2004, 31: 932-939.

3. Varoquaux A, Rager $O$, Poncet $A$ et al. Detection and quantification of focal uptake in head and neck tumours:18F-FDG PET/MR versus PET/CT. Eur J Nucl Med Mol Imaging 2014; 41: 462-475.

4. Kuhn F, Crook D, Mader C, Appenzeller P, von Schuthess GK, Schmid DT. Discrimination and anatomical mapping of PET-positive lesions: comparison of CT attenuation-corrected PET images with coregistered MR and CT images in the abdomen. Eur J Nucl Med Mol Imaging 2013; 40: 44-51.
5. Lin EC, Alavi A (Eds). PET and PET/CT A Clinical Guide. New York: Thieme 2005.

6. Antoch G, Saoudi N, Kuehl H et al. Freudenberg LS: Accuracy of whole-body dual-modality fluorine-18-2-fluoro-2-deoxy-D-glucose positron emission tomography and computed tomography (FDG-PET/CT) for tumor staging in solid tumors: comparison with CT and PET. J Clin Oncol 2004; 22 : 4357-4368.

7. Cohen MS, Arslan N, Dehdashti F et al. Risk of malignancy in thyroid incidentalomas indentified by fluorodeoxyglucose-positron emission tomography. Surgery 2001; 130: 941-946. 
8. Kim TY, Kim WB, Ryu JS, Gong G, Hong SJ, Shong YK. 18F-fluorodeoxyglucose uptake in the thyroid from positron emission tomogram (PET) for evaluation in cancer patients: high prevalence of malignancy in thyroid PET incidentaloma. Laryngoscope 2005; 115: 1074-1078.

9. Nakomoto Y, Tatsumi M, Hammoud D, Cohade C, Osman MM, Wahl RL: Normal FDG distribution patterns in the head and neck: PET/CT evaluation. Radiology 2005; 234: 879-885.
10. Chu QD, Connor MS, Lilien DL, Johnson LW, Turnage RH, Li BD. Positron emission tomography (PET) positive thyroid incidentaloma: the risk of malignancy observed in a tertiary referral center. Am Surg 2006; 72: 272-275.

11. Van den Bruel A, Maes A, De Potter T et al. Clinical relevance of thyroid fluoro-deoxy- glucose-whole body positron emission tomography incidentaloma. J Clin Endocrinol Metab 2002; 87: 1517-1520. 